\title{
Les techniques de régulation mises en œuvre sur le canal et les réseaux de la ville de Marseille
}

\author{
J. Bourgeois, E. Rouanet \\ Société des Eaux de Marseille, 25, rue Edouard Delanglage 13006 Marseille
}

\section{Introduction}

Une régulation n'est pas une fin en soi.

Les régulations adoptées par la Société des Eaux de Marseille ont trois objectifs :

- gérer avec le minimum de pertes les flux d'eau du canal ;

- apporter à chaque station de traitement une eau brute d'une qualité aussi bonne et aussi constante que possible pour faciliter les opérations de potabilisation ;

- garder sur les réseaux de distribution une qualité d'eau irréprochable tout en minimisant le nombre de fuites.

L'alimentation en eau de l'agglomération marseillaise peut se résumer en :

- un canal qui dérive les eaux de la Durance et traverse la totalité du département des Bouches-du-Rhône ;

— une adduction complémentaire et de secours à partir du Verdon ;

- trois usines principales de potabilisation (Ste Marthe, Vallon Dol, St Barnabé) d'où partent les réseaux de desserte des abonnés, réseaux essentiellement gravitaires.

\section{Description et fonctionnement des ouvrages prin- cipaux}

\subsection{Le canal de Marseille}

Le canal de Marseille dérive les eaux de la Durance à partir du canal E.D.F. au niveau de la chute de St Estève. Capable de transiter $17 \mathrm{~m}^{3} / \mathrm{s}$ pour une dotation d'été de $15,118 \mathrm{~m}^{3} / \mathrm{s}$, le canal d'abord Est Ouest traverse ensuite le département des Bouches-du-Rhône suivant un tracé Nord Sud.

Les éléments caractéristiques de ce canal dans sa partie amont sont les suivants :

- en tête, le bassin de St Christophe $\left(2 \mathrm{Mm}^{3}\right)$ permettant une décantation des eaux ;

- un bassin intermédiaire de Réaltort $\left(1 \mathrm{Mm}^{3}\right)$;

- des vannes de sectionnement couplées à des vannes de vidange permettant la constitution "de 6 biefs ".
Dans la partie aval (à partir de Réaltort), le canal comporte une Branche-Mère aboutissant à La Marionne (Est de Marseille) pour se subdiviser en trois dérivations :

- Camoins/Aubagne - La Ciotat

- La Barasse/Montredon

- St Barnabé.

L'eau circule à une vitesse moyenne de $0,8 \mathrm{~m} / \mathrm{s}$ et son fonctionnement est caractérisé par un régime d'hiver (pas d'arrosage) environ $600000 \mathrm{~m}^{3} / \mathrm{j}$ et un régime d'été, environ $950000 \mathrm{~m}^{3} / \mathrm{j}$ au départ de St Estève.

\subsection{Les clients du canal}

A) Les communes diverses le long du canal qui, soit individuellement, soit regroupées en syndicat (SIOM) sont dotées de stations de potabilisation.

B) La Ville de Marseille et les communes du périmètre.

C) Des industriels au niveau de l'étang de Berre.

D) Des arrosants agriculteurs aussi bien sur l'amont que sur l'aval.

\subsection{Les contraintes de gestion du canal}

A) La longueur du canal, la faible vitesse de transit et les clients répartis sur l'ensemble du tracé.

B) Son âge. Conçu entre 1825 et 1845 , ce canal a nécessité de gros travaux de modernisation durant les 30 dernières années :

- mise en place des vannes de sectionnement et de vidange automatisées et télécommandées pour certaines d'entre elles ;

- exhaussement et alésage de souterrains pour uniformiser la portance à $17 \mathrm{~m}^{3} / \mathrm{s}$ dans la partie amont ;

- travaux de confortement de la cuvette à l'occasion de chômages biannuels.

C) Les exhutoires souvent insuffisants

Sur la partie amont, les exhutoires des vannes de vidange, au nombre de six sur l'ensemble du tracé, sont confortables mais ne peuvent, en général, pas accepter la totalité du débit du canal. 


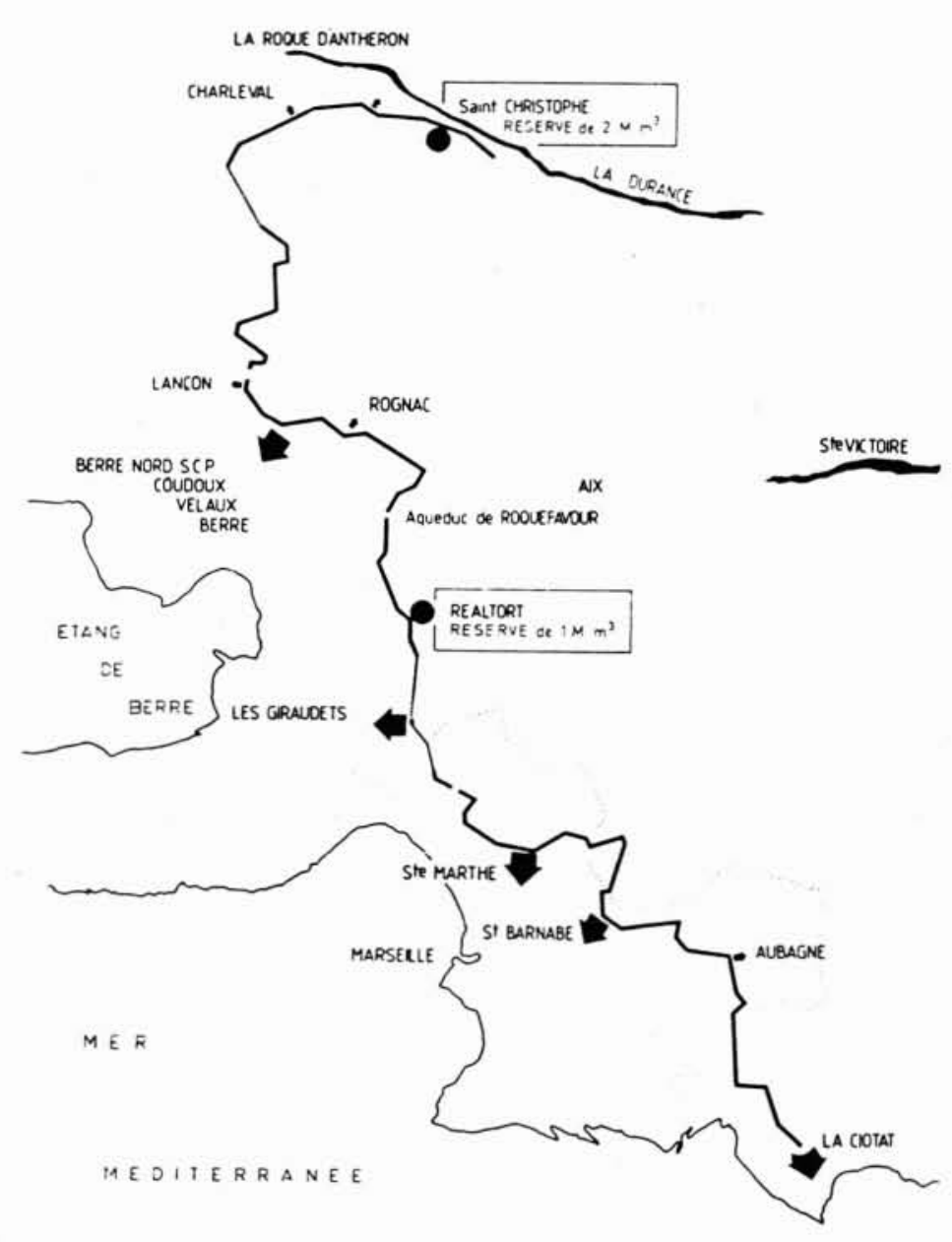

Sur la partie aval, compte-tenu de l'urbanisation, les exhutoires utilisés sont nettement sous dimensionnés et souvent l'objet de limitation de rejet.

D) L'importance des consommations domestiques. Bien que le canal de Marseille desserve une grande diversité d'utilisateurs, les usages domestiques représentent plus de la moitié du débit.

\subsection{Les points forts}

A) Des réserves très importantes

Au niveau du canal lui-même :

- le bassin de St Christophe: $2 \mathrm{Mm}^{3}$ dont $0,4 \mathrm{Mm}^{3}$ mobilisables gravitairement constitue une réserve de tête exceptionnelle ;

- le bassin de Réaltort représentant un volume mobilisable de $1 \mathrm{Mm}^{3}$ (pour un volume transité inférieur à $1 \mathrm{Mm}^{3}$ par jour) permet d'absorber les variations de débits constatées d'un jour sur l'autre.
Au niveau des gros clients intermédiaires:

- Ste Marthe, la principale usine de Marseille fournissant $280000 \mathrm{~m}^{3} / \mathrm{j}$ d'eau filtrée est dotée d'un bassin d'eau brute de $450000 \mathrm{~m}^{3}$;

- l'usine de St Barnabé (100000 $\mathrm{m}^{3} /$ jour) comporte également un décanteur de $145000 \mathrm{~m}^{3}$.

Au niveau des clients d'extrémité :

Les communes de Cassis et de La Ciotat, extrémités de la principale dérivation du canal de Marseille, comportent des réserves respectivement de 16000 et $60000 \mathrm{~m}^{3}$, soit près de 4 jours de consommation.

\section{B) Des arrosages à débit constant}

Les arrosages sont distribués tout le long du canal à tour de rôle avec un débit constant. Dans ces conditions, les débits appelés à ce titre sont bien connus, variant très peu d'un jour à l'autre et quasiment pas dans la journée.

Les refus d'eau sont très rares, la facturation de ces arrosages distribués du $1^{\text {er }}$ avril au 30 septembre est établie au module et non pas au $\mathrm{m}^{3}$.

\section{La régulation du canal au niveau des flux d'eau}

Tenant compte des éléments évoqués ci-dessus, le fonctionnement du canal est assuré de façon différente dans la partie en amont de Réaltort ou dans la partie aval.

\subsection{La régulation amont (entre St Christophe et Réaltort)}

Pour un régime donné (hiver ou été), les variations de débits prévisibles appelés dans cette partie sont statistiquement bien connues et faibles $\left(0,7 \mathrm{~m}^{3} / \mathrm{s}\right)$ par rapport au débit transité par le canal $\left(12 \mathrm{~m}^{3} / \mathrm{s}\right)$.

Le régime adopté pour le transit de l'eau consiste donc à garder le plan d'eau de St Christophe au maximum de sa capacité et d'utiliser le bassin de Réaltort comme tampon.

L'expérience montre que sur ces bases, l'exploitant peut, même en été, rester plus de 10 jours sans changer les consignes de débit au départ de St Christophe.

Chaque bief est doté d'équipement de régulation automatique pour prévenir les conséquences des accidents les plus probables qui sont la rupture d'une berge ou une obstruction dans un souterrain.

Un automatisme assure la commande des vannes du canal en fonction des consignes d'exploitation fixées à l'avance et qui peuvent être modifiées à partir du centre de contrôle par liaison radio.

\subsection{La régulation aval (mise en service en avril 1991)}

Le principe en est basé sur l'utilisation du bassin de St Barnabé $\left(145000 \mathrm{~m}^{3}\right)$ comme réserve de flanc:

- pour permettre à ce réservoir "de battre " sans géner le fonctionnement de l'usine de potabilisation, il a été 
nécessaire de mettre en place des pompes d'exhaure à débit variable et modifier très sensiblement le génie civil des floculateurs situés en tête du bassin ;

- pour une journée donnée, le débit qui part de Réaltort est constant, les prises non dotées de réserves : Giraudets, Berre Sud prélèvent des débits variables, les prises dotées de réserves: Ste Marthe... gardent un débit fixe.

A l'extrémité du canal maître, au lieu-dit La Marionne, point de départ des deux dérivations principales, il est envoyé un débit constant vers Aubagne et La Ciotat, le solde du débit est dirigé vers St Barnabé, qui constate sur le plan d'eau du bassin :

- des variations cycliques dans la journée en fonction de la demande des réseaux d'eau filtrée;

- des variations journalières qui servent de base à la régulation ;

Les variations de débit qui peuvent représenter un maximum de $50000 \mathrm{~m}^{3} /$ jour (pour un volume journalier de $500000 \mathrm{~m}^{3}$ ) sont donc très facilement absorbées par les $145000 \mathrm{~m}^{3}$ du bassin de St Barnabé.

Chaque matin, les volumes prélevés par les diverses prises et la variation du plan d'eau de St Barnabé sont entrés dans le programme informatique qui établit le débit à mettre en ligne à partir de Réaltort, le centre de contrôle effectuant les manœuvres à distance qui en résultent.

La prochaine étape pourra être, lorsque l'ensemble des liaisons sera terminé, la manœuvre automatique des vannes de Réaltort à partir des éléments enregistrés à St Barnabé.

\subsection{Les résultats de la régulation du canal}

La mise en application de notre régulation aval a coincidé avec les périodes délicates des années de sécheresse 1989 et surtout 1990, périodes pendant lesquelles nous avons été conduits à réaliser un maximum d'économies. Le tableau ci-dessous résume les résultats obtenus.

Entre 1989 et 1990, la régulation du canal se traduit par une économie brute chiffrée à $9 \mathrm{Mm}^{3}$ qui mériterait d'être corrigée par des plus et des moins résultant d'autres mesures.

\section{La régulation pour la gestion de la qualité}

Pour un exploitant, l'obtention de la qualité et la conservation de cette qualité est un élément essentiel.

\subsection{Au niveau du canal}

Pour la Société des Eaux de Marseille, le fonctionnement du canal doit fournir aux diverses prises une eau de bonne qualité et aussi constante que possible malgré les variations importantes de cette qualité au niveau de la prise de St Estève.

En effet, les eaux de Durance restent très sensibles aux orages fréquents dans la région même si les ouvrages E.D.F. ont notablement amélioré la situation.

Pour ce faire, le bassin décanteur de St Christophe et les aménagements mis en place et tête de ce bassin constituent un maillon important :

- pour la détection de la pollution : tests poissons, mesures de la turbidité, du pH, des chlorures, de la température et du carbonne organique total ;

- pour traiter les eaux turbides: utilisation du chlorure ferrique dès que la turbidité dépasse $50 \mathrm{NTU}$ et d'autres réactifs : bioxyde de chlore, amidon, charbon actif en fonction des événements.

Cet ouvrage de décantation, remarquable dans sa conception, a un rendement très intéressant ( $70 \%$ sur les matières en suspension).

Dans le même but, le bassin de Réaltort est équipé d'une injection de chlorure ferrique mise en œuvre à la demande et d'un test poisson.

\subsection{La chaîne des informations}

La connaissance de la qualité de l'eau sur le canal : turbidité et température, intéresse le gestionnaire de chaque usine; dans le même esprit, le responsable du canal doit connaître en temps réel ce qui se passe dans les usines. C'est l'objet de la chaîne d'informations centralisée sur un poste déporté du centre de contrôle.

Cette chaîne d'informations établie sur réseau Transfix apporte une grande souplesse au niveau de décision. Elle devrait permettre d'apporter une conclusion à l'étude en cours qui cherche à établir l'intérêt de renforcer le prétraitement sur le canal, d'une part au niveau de St Christophe, d'autre part à la sortie de Réaltort afin de diminuer les taux de chlorure ferrique ou le WAC utilisés dans les diverses usines.

\subsection{La modernisation des stations de traitement}

Après la mise à place des automatismes dans les principales fonctions du traitement de l'eau, l'effort a porté sur la

\begin{tabular}{|c|c|c|c|c|c|}
\hline \multicolumn{6}{|c|}{ Prélèvements au départ de St Christophe } \\
\hline \multicolumn{2}{|c|}{1989} & \multicolumn{2}{|c|}{1990} & \multicolumn{2}{|c|}{1991} \\
\hline Juillet & Août & Juillet & Août & Juillet & Août \\
\hline $29,5 \mathrm{Mm}^{3}$ & $29 \mathrm{Mm}^{3}$ & $29 \mathrm{Mm}^{3}$ & $26 \mathrm{Mm}^{3}$ & $27 \mathrm{Mm}^{3}$ & $26 \mathrm{Mm}^{3}$ \\
\hline
\end{tabular}


régulation au niveau des produits de traitement prenant en compte la température, la turbidité de l'eau du canal, les débits appelés, le résiduel de chlore ou d'ozone...

Les boucles de régulation gèrent le dosage des adjuvants, le chlore en préchloration ainsi que le chlore et l'ozone en traitement final.

\subsection{La gestion unifiée}

Des programmes communs pour toutes les usines ont permis de regrouper les informations dans l'usine principale de Marseille à Ste Marthe, seule usine à l'heure actuelle où des agents travaillent en $3 \times 8$. Ce sont les agents de quarts qui peuvent télécommander les 6 usines de l'agglomération marseillaise ou faire appel, si nécessaire, au personnel d'astreinte.

\section{La régulation des réseaux principaux d'eau potable}

Sur les réseaux principaux essentiellement gravitaires à Marseille, la Société des Eaux a adopté deux types de régulation : celle de débit et celle de pression qui consistent à maintenir constant soit un débit, soit une pression en aval de l'organe régulateur.

\subsection{Les objectifs de ces régulations}

\section{Régulation de débit:}

- provoquer le marnage des réservoirs d'extrémité afin d'éviter, en particulier, une stagnation de l'eau pouvant entraîner la dégradation de sa qualité ;

- éviter, lorsque les réserves des usines de traitement sont insuffisantes, de devoir ajuster le débit de production aux besoins instantanés du réseau de distribution entraînant, en général, une dégradation de l'eau produite.

\section{Régulation de pression :}

- supprimer les variations brutales de pression génératrices de ruptures de canalisation et donc accroître la longévité du réseau ;

- réduire les volumes perdus par les fuites cachées ;

- favoriser le marnage des réservoirs d'extrémité car la perte de charge créée par le régulateur diminue le débit nourricier du réseau ;

- accroître le confort des abonnés en compensant les effets des consommations extrêmes.

\subsection{Moyens mis en auvre}

Les deux types de régulation sont assurés par des équipements identiques, à savoir une vanne spécifique destinée à effectuer la régulation associée à un automate programmable. Les vannes utilisées sont des Monovars de Neyrtec et les automates programmables, soit des APRIL 5000, soit des Moduvars de Neyrtec.
Il suffit de programmer l'automate pour obtenir le type de régulation désiré.

Les installations sont reliées au système de télégestion du réseau de distribution, permettant entre autre la modification à distance de la valeur des paramètres de consigne.

En ce qui concerne la régulation de débit, l'expérience montre, d'une part qu'il faut prévoir l'installation d'un débitmètre précis (type ultrasons ou électromagnétique) bien qu'à priori les mesures de pression en amont et en aval de l'organe de régulation permettent le calcul du débit et, d'autre part qu'il est indispensable de disposer d'une sécurité permettant de passer automatiquement en régulation de pression au cas où cette dernière viendrait à dépasser une valeur de consigne prédéterminée.

La valeur de consigne de sécurité peut être, soit un seuil programmé sur l'automate, soit un contact sec délivré à l'automate par un manostat.

\subsection{Résultats observés}

Expérience de la régulation de débit: A Marseille, l'objectif unique de ce type de régulation est de provoquer le marnage de certains réservoirs d'équilibre dont la capacité est largement dimensionnée par rapport aux besoins actuels de leur zone d'influence. L'objectif peut être considéré comme atteint, des traces de chlore libre résiduel étant décelées dans l'eau de ces réservoirs.

Expérience de la régulation de pression: La mise en service des régulateurs remonte aux années 1982-1983. Outre une diminution du nombre de réclamations d'abonnés soumis auparavant à d'importantes variations de pression, une réduction notoire du nombre annuel de fuites sur le réseau et les branchements a été observée : 3100 fuites en 1981, 1800 en 1984 et 1400 depuis 1989 (à noter que les années 1985, 1986 et 1987 n'ont pas été significatives car marquées par des gels rigoureux ayant provoqué de nombreuses ruptures de canalisations).

\section{Conclusions}

Réguler pour assurer la qualité de l'eau, opération primordiale et nécessitant certaine aisance dans les flux d'eau peut paraître contradictoire avec la régulation des débits.

Le contexte de l'alimentation en eau de la Ville de Marseille: réserves importantes - débits relativement constants - réseaux principaux gravitaires, permet de privilégier la fonction qualité en restant rigoureux au niveau des déversements et des pertes.

Les nouveaux outils (automates programmables) et les liaisons récentes permettant de relier les gestionnaires du canal, des diverses usines et des réseaux avec des langages et données communes, constituent les bases essentielles des progrès qui ont pu être développés ces dernières années. 